\section{Inactivation du chromosome $X$ et pluripotence}

Pablo Navarro
Unité de génétique

moléculaire murine, Département de biologie du développement, Institut Pasteur,

28, rue du Docteur Roux, 75015 Paris, France. pablo.navarro@ed.ac.uk

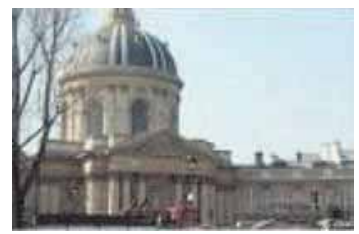

> L'inactivation d'un chromosome $X$ (ICX) chez les femelles (XX) est le processus qui permet de compenser le niveau d'expression des gènes $d u$ chromosome $X$ entre les mammifères mâles (XY) et femelles (XX) [1]. Chez la souris, il existe deux formes distinctes d'ICX: la première caractérise les cellules de l'embryon préimplantatoire et des annexes extraembryonnaires, et affecte exclusivement le chromosome $X$ d'origine paternel $(X p)$; en revanche, dans les cellules de l'embryon postimplantatoire ainsi que dans les cellules adultes, le deuxième processus d'inactivation peut cibler aussi bien le chromosome $X$ d'origine paternelle $(X p)$ que d'origine maternelle $(X m)$. À un certain moment de l'embryogenèse précoce, les mécanismes d'inactivation de I'X doivent donc être reprogrammés pour passer d'une inactivation systématique de I'Xp à une inactivation aléatoire (Figure 1).

Cette transition s'effectue dans les cellules pluripotentes de la masse cellulaire interne $(\mathrm{MCl})$ du blastocyste, où le $X p$ inactif est réactivé $[2,3]$. Ceci aboutit à la présence de deux chromosomes $X$ actifs dans chaque cellule de la $\mathrm{MCl}$. L'inactivation aléatoire se met en place lorsque ces cellules engagent leur processus de différenciation pour former les trois lignages primordiaux de l'embryon (ectoderme, endoderme, mésoderme). Par la suite, toutes les cellules issues des premières cellules ayant inactivé soit le $X p$ soit le $X m$ conservent ce profil d'inactivation, générant ainsi des individus femelles mosaïques composés de clones cellulaires ayant inactivé soit l'un soit l'autre des deux chromosomes X [17]. La seule exception à cette stabilité de l'inactivation aléatoire intervient dans la lignée germinale femelle (Figure 1): la ré-acquisition de propriétés de pluripotence nécessaire à la génération des gamètes s'accompagne à nouveau d'une réactivation de I'X inactif [4]. Hormis les cellules pluripotentes de la $\mathrm{MCl}$ et de la lignée germinale, toutes les cellules femelles sont donc caractérisées par la présence d'un chromosome $X$ inactif. Cette observation a été consolidée par l'étude in vitro de différents modèles de cellules pluripotentes (Figure I) : ainsi, la différenciation des cellules souches embryonnaires (cellules ES) s'accompagne d'une ICX aléatoire; réciproquement, lorsqu'une cellule somatique est reprogrammée en cellule pluripotente par différentes techniques (iPS ou induced pluripotent stem cells), alors I'X inactif est réactivé $[5,6]$.

Bien que le premier modèle proposant un contrôle au cours du développement du chromosome $X$, qui associait son inactivation à la perte de la pluripotence, ait été énoncé dès 1978 [7], les mécanismes sous-jacents sont longtemps restés mystérieux. Pour les comprendre, il a tout d'abord fallu élucider les facteurs moléculaires clés contrôlant chacun de ces processus, ICX et pluripotence.

\section{Xist, un acteur incontournable}

Concernant l'inactivation du chromosome $X$, la découverte fondamentale du gène Xist en 1991 a représenté une avancée majeure dans le domaine [8, 9]. Lié au chromosome $X$, le gène Xist produit un ARN non codant de grande taille possédant la propriété unique de recouvrir le chromosome $X$ qui le produit pour enclencher le processus de répression [1]. Dans les cellules pluripotentes femelles de l'embryon, issues de la $\mathrm{MCl}$ et de la lignée germinale, ainsi que dans les cellules ES, l'expression de Xist est réprimée à partir des deux chromosomes $X$ actifs. En revanche, lorsque la pluripotence est perdue au cours des processus de différenciation, l'expression de Xist se met en place à partir d'un seul chromosome $X$, le futur $X$ inactif (Figure 1 ). Déterminer les mécanismes responsables de l'extinction de Xist dans les cellules pluripotentes semble donc important pour appréhender la régulation développementale de I'ICX.

L'état de pluripotence, quant à lui, est atteint et maintenu par l'action concertée de trois facteurs de transcription, Nanog, Oct4 et Sox2, dont l'expression simultanée est spécifique des cellules pluripotentes [10]. D'une part, ils agissent de concert pour réprimer les gènes du développement et donc pour maintenir l'état indifférencié; d'autre part, ils activent simultanément les gènes nécessaires à l'autorenouvellement de cet état indifférencié et pluripotent. Selon le scénario communément admis jusqu'en 2008, la régulation de Xist et de I'ICX apparaissait comme un effet secondaire de l'action de Nanog, Oct4 et Sox2 lors de l'établissement du phénotype pluripotent. En effet, l'acquisition de la pluripotence s'accompagne d'une reprogrammation épigénétique à grande échelle qui pourrait prendre en charge la régulation de I'ICX, sans qu'aucun facteur spécifique n'y soit directement impliqué. 


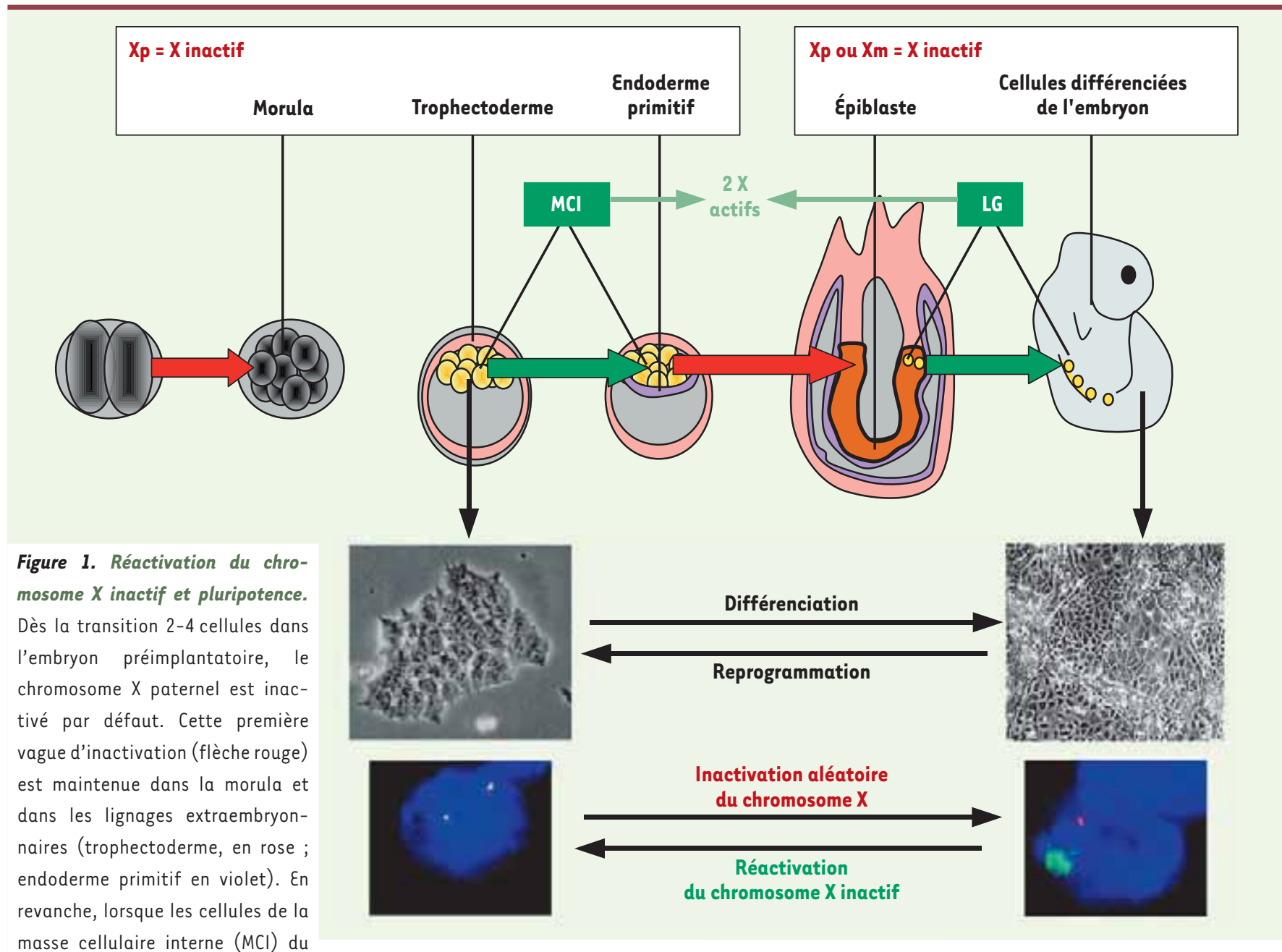

blastocyste acquièrent la pluripotence, alors l'Xp inactif est spécifiquement réactivé (flèche verte). Par la suite, les cellules de la MCl se différencient pour former les tissus de l'embryon à proprement parler, et l'inactivation aléatoire se met en place (flèche rouge). Le chromosome X inactif aléatoirement choisi ne sera réactivé (flèche verte) que dans la lignée germinale (LG). Dans l'embryon, l’X inactif est donc réactivé uniquement dans les compartiments pluripotents. In vitro, la différenciation des cellules ES est accompagnée par l'inactivation aléatoire, mise en place après le recouvrement d'un seul $X$ par l'ARN Xist (visualisé ici par la technique d'ARN FISH ; en vert, Xist, en rouge un gène du chromosome X qui s'exprime, dans les cellules différenciées, exclusivement à partir de I'X non recouvert par Xist). Le mécanisme inverse peut être observé lorsqu'une cellule somatique est reprogrammée en cellule pluripotente. Dans les cellules pluripotentes, l'absence d'inactivation est due, au moins en partie, à l'absence d'expression de Xist.

Xist : une cible directe

des gènes clés de la pluripotence

Contrairement à cette idée, il a tout d'abord été montré que Xist est transcriptionnellement inactif dans les cellules $\varepsilon S$ indifférenciées [11], et que cette répression semble indépendante de la structure chromatinienne de ce gène [12]. II y aurait donc des régulateurs spécifiques responsables de l'extinction de Xist dans les cellules ES. Par ailleurs, dans la lignée germinale, la répression de Xist s'instaure avant même la reprogrammation épigénétique globale [13], et tout comme lors de la formation de la $\mathrm{MCl}$ [2], l'extinction de Xist suit de très près l'expression de Nanog. Ce facteur de transcription pourrait donc être directement impliqué dans la répression transcriptionnelle de Xist. La démonstration de la fixation de Nanog au niveau de l'intron 1 du gène Xist dans les cellules ES, ainsi que celle de la perte de cette fixation lors de l'initiation de l'ICX [14], semble confirmer cette hypothèse. Cette interaction directe a été également observée entre Xist et 0ct4 et Sox2, les deux autres piliers de l'état pluripotent [14]. Ces résultats révèlent donc vrai- semblablement l'existence d'un lien moléculaire intime entre pluripotence et régulation de Xist (Figure 2), bien au-delà de ce qui avait été anticipé. De plus, dans des cellules mâles transgéniques où la perte de ces trois facteurs peut être induite, une surexpression rapide et importante de l'expression de Xist, compatible avec la mise en place de l'ICX, a été démontrée [14]. Puisque dans les cellules mâles Xist n'est jamais surexprimé, c'est la preuve d'un lien direct entre l'expresssion de Nanog, 0ct4 et Sox2 et le maintien de Xist à l'état silencieux, leur extinction au cours de la différenciation représentant le signal 


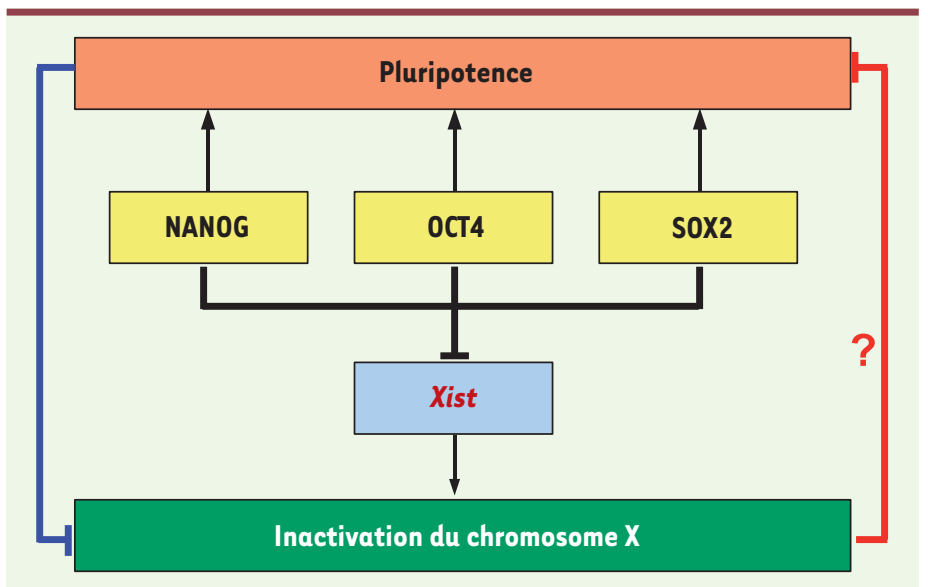

Figure 2. Réseau transcriptionnel couplant les régulations de la pluripotence et de l'inactivation de l'X. II était admis que le phénotype pluripotent, sous le contrôle de trois facteurs de transcription (Nanog, 0ct4 et Sox2), entraînait l'extinction de Xist et la réactivation de I'X inactif par les mécanismes épigénetiques qui agissent sur l'ensemble du génome (ligne bleue). Cependant, il s'est avéré que les mêmes facteurs qui contrôlent la pluripotence sont aussi directement responsables de l'extinction transcriptionnelle de Xist dans les cellules ES. Le couplage entre ces deux processus épigénetiques est donc strict et génétiquement régulé. Ceci suggère que la réactivation du chromosome $X$ inactif pourrait être une étape nécessaire pour générer ou consolider la pluripotence (ligne rouge). moléculaire qui couple I'ICX à la perte de la pluripotence $[17,18]$. Néanmoins, ces conclusions devront être confirmées par des expériences analysant les conséquences de la délétion génétique de l'intron 1 du gène Xist.

\section{Deux $X$ actifs, nécessité ou} conséquence de la pluripotence?

Ces résultats font de Nanog, 0 ct4 et Sox2 les répresseurs majeurs de Xist dans les cellules ES indifférenciées (Figure 2). Ils constituent en cela le premier élément de réponse aux diverses observations qui, depuis 30 ans, ont mené à ces deux constats: l'absence d'inactivation du chromosome $X$ est une caractéristique intrinsèque des cellules pluripotentes, et I'ICX aléatoire s'instaure par défaut au cours de la différenciation cellulaire [15]. Ils permettent aussi de proposer un scénario moléculaire permettant d'expliquer la dynamique de régulation de l'inactivation lors des différentes transitions développementales mettant en jeu l'acquisition et la perte de la pluripotence (Figure I).

Le couplage moléculaire mis en évidence entre pluripotence et inactivation du chromosome $X$ (Figure 2) soulève des questions importantes et parfois provocantes, dans le domaine de la pluripotence. En effet, il est admis que seuls les gènes nécessaires au phénotype pluripotent sont contrôlés directement et simultanément par le triumvirat de facteurs Nanog, Oct4 et Sox2. Ceci suggère que l'extinction de Xist, et donc la présence de deux $X$ actifs dans les cellules femelles pourrait être une nécessité, plutôt qu'une conséquence, de la pluripotence. Ce serait là une différence inattendue entre cellules mâles et femelles, à moins qu'un deuxième système de compensation n'agisse, qui permette la surexpression du seul chromosome X chez les mâles [16].

$X$ inactivation: a direct

link with the pluripotent state?

\section{Pablo Navarro, 30 ans}

Après avoir quitté l'Espagne à l'âge de 18 ans pour une formation en classe préparatoire, Pablo Navarro intègre l'École Normale Supérieure de Cachan. Lors d'un stage de maîtrise, c'est le coup de foudre avec l'inactivation du chromosome $X$ et le laboratoire de Philip Avner (Institut Pasteur, génétique moléculaire murine), où il reste pour son $D E A$, sa thèse et son premier stage postdoctoral. À Edimbourg, Pablo Navarro se consacre maintenant à la pluripotence avec lan Chambers du Institute for Stem Cell Research, dirigé par le père du célèbre mouton cloné Dolly.

\section{CONFLIT D'INTÉRÊTS}

L'auteur déclare n'avoir aucun conflit d'intérêts concernant les données publiées dans cet article.

\section{RÉFÉRENCES}

1. Heard $\varepsilon$, Disteche CM. Dosage compensation in mammals: fine-tuning the expression of the $X$ chromosome. Genes Dev $2006 ; 20: 1848-67$
2. Mak W, Nesterova TB, de Napoles M, et al. Reactivation of the paternal $X$ chromosome in early mouse embryos. Science 2004 ; 303 : 666-9.

3. Okamoto I, Otte AP, Allis CD, Reinberg D, Heard $\varepsilon$. Epigenetic dynamics of imprinted $X$ inactivation during early mouse development. Science 2004 ; 303 : 644-9.

4. Gartler SM, Andina R, Gant N. Ontogeny of X-chromosome inactivation in the female germ line. Exp Cell Res 1975 ; $91: 454-7$

5. Takagi N, Yoshida MA, Sugawara O, Sasaki M. Reversal of Xinactivation in female mouse somatic cells hybridized with murine teratocarcinoma stem cells in vitro. Cell 1983; $34:$ 1053-62

6. Stadtfeld M, Maherali N, Breault DT, Hochedlinger K. Defining molecular cornerstones during fibroblast to iPS cell reprogramming in mouse. Cell Stem Cell 2008; $2: 230-40$.

7. Monk M, Harper MI. Sequential X chromosome inactivation coupled with cellular differentiation in early mouse embryos. Nature $1979 ; 281$ : 311-3.

8. Borsani G, Tonlorenzi R, Simmler MC, et al. Characterization of a murine gene expressed from the inactive $X$ chromosome. Nature 1991; 351: 325-9.

9. Brown C), Ballabio A, Rupert JL, et al. A gene from the region of the human $X$ inactivation centre is expressed exclusively from the inactive $X$ chromosome. Nature $1991 ; 349$ : 38-44.

10. Chambers I, Tomlinson SR. The transcriptional foundation of pluripotency. Development 2009; 136 : 2311-22.

11. Navarro P, Pichard S, Ciaudo C, et al. Tsix transcription across the Xist gene alters chromatin conformation without affecting Xist transcription: implications for $X$-chromosome inactivation. Genes Dev $2005 ; 19$ : 1474-84.

12. Navarro P, Page DR, Avner P, Rougeulle C. Tsix-mediated epigenetic switch of a CTCF-flanked region of the Xist promoter determines the Xist transcription program. Genes Dev $2006 ; 20: 2787-92$.

13. De Napoles M, Nesterova T, Brockdorff N. Early loss of Xist RNA expression and inactive $X$ chromosome associated chromatin modification in developing primordial germ cells. PLoS One 2007 ; 2 : e860

14. Navarro P, Chambers I, Karwacki-Neisius V, et al. Molecular coupling of Xist regulation and pluripotency. Science 2008; 321: 1693-5.

15. Navarro $P$, Avner $P$. When $X$-inactivation meets pluripotency: an intimate rendezvous. FEBS Lett $2009 ; 583$ : 1721-7.

16. Nguyen DK, Disteche CM. Dosage compensation of the active $X$ chromosome in mammals. Nat Genet 2006 ; $38: 47-53$

17. Augui $S$, Heard $\varepsilon$. Inactivation du chromosome $X$ : comment une cellule sait compter jusqu'à deux X. Med Sci (Paris) $2008 ; 24$ : 584-5.

18. Vigneau S, Clerc P. Sans Tsix, les mâles aussi inactivent leur chromosome X. Med Sci (Paris) $2006 ; 22: 926-8$. 